\title{
Long-Term Stability of $n$-Alkane-in-Water Pickering Nanoemulsions: Effect of Aqueous Solubility of Droplet Phase on Ostwald Ripening
}

\author{
Kate L. Thompson,* Matthew J. Derry, ${ }^{\circledR}$ Fiona L. Hatton, ${ }^{\circledR}$ and Steven P. Armes*(-) \\ Department of Chemistry, University of Sheffield, Dainton Building, Brook Hill, Sheffield, South Yorkshire S3 7HF, U.K.
}

Supporting Information

ABSTRACT: High-pressure microfluidization is used to prepare a series of oil-in-water Pickering nanoemulsions using sterically-stabilized diblock copolymer nanoparticles as the Pickering emulsifier. The droplet phase comprised either $n$-octane, $n$-decane, $n$-dodecane, or $n$-tetradecane. This series of oils enabled the effect of aqueous solubility on Ostwald ripening to be studied, which is the primary instability mechanism for such nanoemulsions. Analytical centrifugation (LUMiSizer instrument) was used to evaluate the long-term stability of these Pickering nanoemulsions over time scales of weeks/months. This technique enables convenient quantification of the fraction of growing oil droplets and confirmed that using $n$-octane (aqueous solubility $=0.66 \mathrm{mg} \mathrm{dm}^{-3}$ at 20

${ }^{\circ} \mathrm{C}$ ) leads to instability even over relatively short time periods. However, using $n$-tetradecane (aqueous solubility $=0.386 \mu \mathrm{g}$ $\mathrm{dm}^{-3}$ at $20^{\circ} \mathrm{C}$ ) leads to significantly improved long-term stability with respect to Ostwald ripening, with all droplets remaining below $1 \mu \mathrm{m}$ diameter after 6 weeks storage at $20^{\circ} \mathrm{C}$. In the case of $n$-dodecane, the long-term stability of these new copolymerstabilized Pickering nanoemulsions is significantly better than the silica-stabilized Pickering nanoemulsions reported in the literature by Persson et al. (Colloids Surf., A, 2014, 459, 48-57). This is attributed to a much greater interfacial yield stress for the former system, as recently described in the literature (see P. J. Betramo et al. Proc. Natl. Acad. Sci. U.S.A., 2017, 114, 1037310378).

\section{INTRODUCTION}

Pickering emulsions can be defined as emulsion droplets that are stabilized by solid particles and have been recognized since the beginning of the last century. ${ }^{1,2}$ Both water-in-oil $(\mathrm{w} / \mathrm{o})^{3-5}$ or oil-in-water $(\mathrm{o} / \mathrm{w})^{6-8}$ Pickering emulsions are widely reported in the literature, with additional recent examples including water-in-water $(\mathrm{w} / \mathrm{w})^{9,10}$ and oil-in-oil $(\mathrm{o} / \mathrm{o})$ Pickering emulsions. ${ }^{11,12}$ Examples of suitable Pickering emulsifiers include silica, ${ }^{6,13}$ titania, ${ }^{14}$ clays, ${ }^{15-18}$ carbon black, $^{19}$ and latex particles; ${ }^{5,7,20}$ in this context, emulsifier efficacy is governed primarily by surface wettability rather than by bulk chemical composition.

Recently, there have been a few literature reports of the preparation of Pickering nanoemulsions. ${ }^{21-23}$ A nanoemulsion comprises very fine droplets that are less than approximately $200 \mathrm{~nm}$ in diameter. ${ }^{24}$ Compared to macroemulsions, nanoemulsions are significantly less susceptible to gravitational creaming or sedimentation. In addition, their much higher surface area is expected to lead to more active formulations for cosmetics, $^{25}$ drug delivery, ${ }^{26}$ food manufacturing, ${ }^{27}$ and agrochemical applications. $^{28}$

To date, most nanoemulsion studies have utilized various surfactants as the emulsifier. ${ }^{29-32}$ Significantly, fewer literature examples involve Pickering nanoemulsions, perhaps because their stabilization requires relatively small nanoparticles

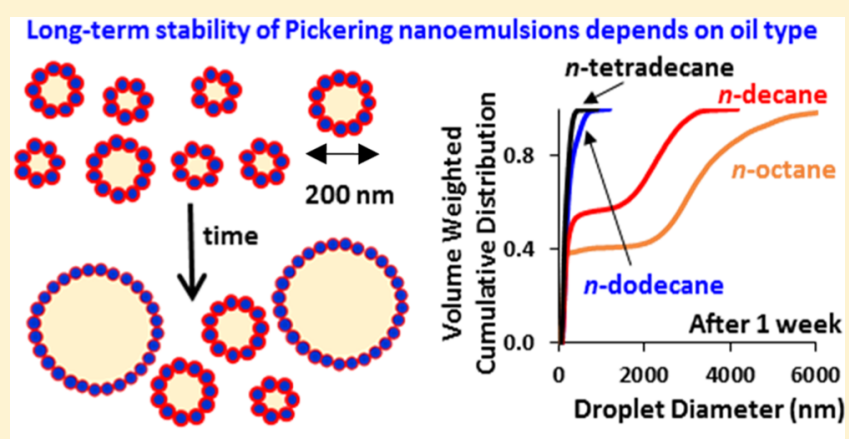

Ostwald ripening of oil droplets monitored by analytical centrifugation (typically at least 5-10 times smaller than the mean droplet diameter) which may not be readily available. ${ }^{21-23,33}$ Nevertheless, Persson et al. ${ }^{21}$ used a high-pressure microfluidizer to prepare o/w Pickering nanoemulsions of between 100 and 200 $\mathrm{nm}$ diameter stabilized using a commercial $7 \mathrm{~nm}$ silica sol. Unfortunately, Ostwald ripening was observed over time periods of hours (for $n$-octane) to weeks (for $n$-tetradecane) when using various $n$-alkanes, even for relatively waterinsoluble $n$-alkanes such as $n$-hexadecane. However, this instability mechanism, which is well-known for nanoemulsions owing to their relatively high surface area, ${ }^{30}$ could be suppressed by employing a highly water-insoluble oil such as squalene.

Using a similar high-pressure microfluidizer setup as that utilized by Persson et al., we recently reported ${ }^{34}$ the preparation of relatively stable $n$-dodecane-in-water Pickering nanoemulsions stabilized by poly(glycerol monomethacrylateblock-2,2,2-trifluoroethyl methacrylate) (PGMA-PTFEMA) diblock copolymer nanoparticles of $25 \mathrm{~nm}$ diameter. Nanoemulsions with mean hydrodynamic droplet diameters as small as $220 \mathrm{~nm}^{35}$ could be prepared that remained stable for up to 4

Received: June 1, 2018

Revised: July 10, 2018

Published: July 12, 2018 
months, as judged by dynamic light scattering (DLS) studies. Herein, we extend this preliminary study by comparing the relative long-term stabilities of $\mathrm{o} / \mathrm{w}$ Pickering nanoemulsions prepared using $n$-octane, $n$-decane, $n$-dodecane, or $n$ tetradecane. This series of four oils was chosen to examine the extent of Ostwald ripening. These nanoemulsions were characterized using three well-established particle-sizing techniques, namely DLS (Malvern Nanosizer), laser diffraction (Malvern Mastersizer), and analytical centrifugation (LUMiSizer instrument).

\section{EXPERIMENTAL SECTION}

Materials. Glycerol monomethacrylate (GMA, 99.8\% purity) was obtained from GEO Specialty Chemicals (Hythe, UK) and was used as received. 2-Cyano-2-propyl benzodithioate, 2,2,2-trifluoroethyl methacrylate (TFEMA), 4,4'-azobis (4-cyanopentanoic acid) (ACVA), fluorescein $O$-methacrylate (FluMA), $n$-dodecane, $n$-octane, $n$-tetradecane, dichloromethane, and deuterium oxide were purchased from Aldrich (UK) and were used as received, unless otherwise stated. Ethanol and dimethylformamide (DMF) were purchased from VWR chemicals (UK), while $n$-decane was purchased from Alfa Aesar (UK).

Synthesis of PGMA $_{48}$ Macro-CTA via RAFT Solution Polymerization in Ethanol. A PGMA 48 macro-CTA (hereafter described as $\mathrm{PGMA}_{48}$ ) was synthesized via RAFT polymerization of glycerol monomethacrylate in ethanol at $70{ }^{\circ} \mathrm{C}$, as described previously. ${ }^{21,30}{ }^{1} \mathrm{H}$ NMR studies indicated a mean DP of 48 via end-group analysis (integrated aromatic RAFT end-group signals at 7.1-7.4 ppm were compared to those of the two oxymethylene protons at 3.5-4.4 ppm). DMF gel permeation chromatography (GPC) studies (refractive index detector) indicated an $M_{\mathrm{n}}$ of $12700 \mathrm{~g}$ $\mathrm{mol}^{-1}$ and an $M_{\mathrm{w}} / M_{\mathrm{n}}$ of 1.17 , with a series of near-monodisperse poly(methyl methacrylate) standards being used for calibration.

Synthesis of PGMA P $_{48}-$ PTFEMA $_{50}$ Diblock Copolymer Nanoparticles via RAFT Aqueous Emulsion Polymerization. $\mathrm{PGMA}_{48}-\mathrm{PTFEMA}_{50}$ diblock copolymer nanoparticles were synthesized as follows: PGMA $_{48}$ macro-CTA $(2.830 \mathrm{~g})$, ACVA (0.020 g, $71.4 \mu \mathrm{mol}$; macro-CTA/ACVA molar ratio $=5.0)$, and water $(52.65$ g, $10 \mathrm{w} / \mathrm{w} \%)$ were weighed into a $100 \mathrm{~mL}$ round-bottomed flask, sealed with a rubber septum and degassed with nitrogen for $30 \mathrm{~min}$. TFEMA ( $2.54 \mathrm{~mL}, 17.8 \mathrm{mmol}$, target DP $=50)$, which had been deoxygenated separately with nitrogen for $15 \mathrm{~min}$, was then added to the solution under nitrogen and immersed in an oil bath set at $70^{\circ} \mathrm{C}$. The reaction solution was stirred for $20 \mathrm{~h}$ to ensure complete TFEMA monomer conversion, and the polymerization was quenched by exposure to air. ${ }^{19} \mathrm{~F}$ NMR spectroscopy analysis of the copolymer dissolved in $d_{6}$-acetone indicated less than $1 \%$ residual TFEMA monomer. DMF GPC studies indicated an $M_{\mathrm{n}}$ of $19100 \mathrm{~g} \mathrm{~mol}^{-1}$ and a $M_{\mathrm{w}} / M_{\mathrm{n}}$ of 1.14 , with a series of near-monodisperse poly(methyl methacrylate) standards being used for calibration.

Preparation of PGMA $_{48}-$ PTFEMA $_{50}$-Stabilized Pickering Macroemulsions Using High-Shear Homogenization. A $\mathrm{PGMA}_{48}-\mathrm{PTFEMA}_{50}$ aqueous dispersion $(8.0 \mathrm{~mL} ; 7.0 \mathrm{w} / \mathrm{w} \%$ solids) was added to a $14 \mathrm{~mL}$ glass vial and homogenized with $2.0 \mathrm{~mL}$ of $n$-dodecane for $2.0 \mathrm{~min}$ at $20{ }^{\circ} \mathrm{C}$ using an IKA Ultra-Turrax T-18 homogenizer with a $10 \mathrm{~mm}$ dispersing tool operating at $15500 \mathrm{rpm}$. The resulting milky oil-in-water emulsion was then analyzed by optical microscopy and laser diffraction. The same protocol was used for the other three $n$-alkanes used in this work.

Preparation of $\mathrm{PGMA}_{48}-\mathrm{PTFEMA}_{50}$-Stabilized Pickering Nanoemulsions Using High-Pressure Microfluidization. A Pickering macroemulsion $(6.0 \mathrm{~mL})$ was further processed using an LV1 Low Volume Microfluidizer Processor (Microfluidics, USA). The pressure was adjusted to $20000 \mathrm{psi}$, and the number of passes through the LV1 was fixed at 10.

Characterization. NMR Spectroscopy. ${ }^{1} \mathrm{H}$ and ${ }^{19} \mathrm{~F}$ NMR spectra were recorded in $d_{6}$-acetone, $\mathrm{D}_{2} \mathrm{O}$, or $\mathrm{CD}_{3} \mathrm{OD}$ using a Bruker AVANCE 400 spectrometer operating at $400 \mathrm{MHz}$.

Gel Permeation Chromatography. Molecular weights and dispersities were assessed using a GPC instrument equipped with a
Varian 290-LC pump injection module, a Varian 390-LC refractive index detector, and two Polymer Laboratories PL gel $5 \mu \mathrm{m}$ mixed-C columns with a DMF mobile phase containing $0.01 \mathrm{M} \mathrm{LiBr}$ operating at $60{ }^{\circ} \mathrm{C}$ with a constant flow rate of $1.0 \mathrm{~mL} \mathrm{~min}{ }^{-1}$. Dimethyl sulfoxide was used as a flow-rate marker, and calibration was achieved using a series of near-monodisperse poly(methyl methacrylate) standards.

Dynamic Light Scattering. Z-average hydrodynamic diameters were determined by DLS at $25^{\circ} \mathrm{C}$ using a Malvern Zetasizer NanoZS instrument at a fixed scattering angle of $173^{\circ}$. Dilute aqueous dispersions $(0.01 \mathrm{w} / \mathrm{w} \%)$ of $\mathrm{PGMA}_{48}-\mathrm{PTFEMA}_{50}$ nanoparticles were analyzed using disposable cuvettes, and the results were averaged over three consecutive runs. The deionized water used to dilute each sample was ultrafiltered through a $0.20 \mu \mathrm{m}$ membrane to remove extraneous dust.

Laser Diffraction. Each macroemulsion was sized using a Malvern Mastersizer 3000 instrument equipped with a hydro EV wet sample dispersion unit, a red $\mathrm{HeNe}$ laser operating at $633 \mathrm{~nm}$ and a lightemitting diode blue-light source operating at $470 \mathrm{~nm}$. The stirring rate was adjusted to $1500 \mathrm{rpm}$ to avoid creaming of the emulsion during analysis. After each measurement, the cell was rinsed three times with deionized water; the glass walls of the cell were carefully wiped with a lens cleaning tissue to avoid cross-contamination, and the laser was aligned centrally to the detector prior to data acquisition.

Analytical Centrifugation (LUMiSizer). Droplet size distributions were assessed using a LUMiSizer analytical photocentrifuge (LUM $\mathrm{GmbH}$, Berlin, Germany) at $20{ }^{\circ} \mathrm{C}$. Measurements were conducted on diluted Pickering nanoemulsions $(1.0 \mathrm{v} / \mathrm{v} \% n$-alkane $)$ in $2 \mathrm{~mm}$ path length polyamide cells at $200 \mathrm{rpm}$ for 200 profiles (allowing $20 \mathrm{~s}$ between profiles), and then the rate of centrifugation was increased up to $4000 \mathrm{rpm}$ for a further 800 profiles. The slow initial rate of centrifugation enabled detection of any larger oil droplets that might be present within the nanoemulsion. The LUMiSizer instrument employs space- and time-resolved extinction profiles technology to measure the intensity of transmitted near-infrared light as a function of time and position over the entire cell length simultaneously. The gradual progression of these transmission profiles contains information on the rate of creaming and, in principle, enables assessment of the droplet size distribution. The nanoemulsion droplet density is an essential input parameter for such analytical centrifugation studies. In the present case, the effective droplet density is somewhat higher than that of the pure $n$-alkane owing to the presence of the adsorbed monolayer of relatively dense $\mathrm{PGMA}_{48}-\mathrm{PTFEMA}_{50}$ nanoparticles. Nevertheless, the true droplet density must lie between that of the pure $n$-alkane and water because visual inspection confirmed that droplet creaming always occurred during the LUMiSizer analysis. The effective density of the nanoparticle-coated $n$-alkane droplets can be estimated by using eqs $1-3$ (see below). One caveat here is that a density distribution is superimposed on the droplet size distribution for each Pickering nanoemulsion. On the basis of our earlier studies, ${ }^{36,37}$ this is expected to lead to an artefactual broadening ${ }^{38}$ of the LUMiSizer droplet size distribution. Moreover, the superimposed density distribution is expected to change over time as the droplet size distribution broadens as a result of Ostwald ripening. Thus, correcting for this secondary effect is rather complex and is considered to be beyond the scope of the present study, which instead focuses on assessing the relative long-term stabilities of Pickering nanoemulsions prepared using four different $n$-alkanes. In view of this, we chose to estimate the effective droplet density from the initial $z$ average DLS diameter recorded for each fresh nanoemulsion, and this value was utilized for all subsequent time-dependent LUMiSizer studies.

Small-Angle X-ray Scattering (SAXS). SAXS data were acquired using a laboratory-based SAXS instrument (Xeuss 2.0, Xenocs, France) equipped with a liquid gallium MetalJet X-ray source (Excillum, Sweden, wavelength $\lambda=0.134 \mathrm{~nm}$ ), with motorized scatterless slits for beam collimation and a Dectris PILATUS 1M pixel detector. The sample-to-detector distance (or camera length) was $2.493 \mathrm{~m}$. SAXS patterns were recorded over a $q$ range of $0.04 \mathrm{~nm}^{-1}<$ $q<3.0 \mathrm{~nm}^{-1}$, where $q(=4 \pi \sin \theta / \lambda)$ is the scattering vector and $\theta$ is 
one-half of the scattering angle. Glass capillaries of approximately 2.0 $\mathrm{mm}$ diameter were used for all samples. Data were reduced using the Foxtrot software package developed by SOLEIL and further analyzed using Irena SAS macros ${ }^{39}$ for Igor Pro.

Calculation of Nanoparticle and Droplet Densities. The $\mathrm{PGMA}_{48}-\mathrm{PTFEMA}_{50}$ nanoparticle density $\left(\rho_{\text {particle }}\right)$ was calculated using eq 1 , as described in our earlier study. ${ }^{36}$

$$
\rho_{\text {particle }}=\frac{\rho_{\text {PTFEMA }} R_{\mathrm{s}}{ }^{3}+\rho_{\text {PGMA }}\left[\left(R_{\mathrm{s}}+2 R_{\mathrm{g}}\right)^{3}-R_{\mathrm{s}}{ }^{3}\right]}{\left(R_{\mathrm{s}}+2 R_{\mathrm{g}}\right)^{3}}
$$

Here, $\rho_{\text {PTFEMA }}$ and $R_{s}$ denote the density and radius of the PTFEMA ${ }_{50}$ core-forming block, and $\rho_{\mathrm{PGMA}}$ and $R_{\mathrm{g}}$ are the density and radius of gyration of the shell component $\left(\mathrm{PGMA}_{48}\right) \cdot 2 R_{\mathrm{g}}$ is equal to the PGMA stabilizer shell thickness. The numerical value of $\rho_{\text {PTFEMA }}$ was taken to be $1.47 \mathrm{~g} \mathrm{~cm}^{-3}$, which is the density of nonsolvated PTFEMA, whereas the highly hydrated PGMA shell was estimated to have a density $\rho_{\mathrm{PGMA}}$ of approximately $1.00 \mathrm{~g} \mathrm{~cm}^{-3} . R_{\mathrm{s}}=6.81 \mathrm{~nm}$ and $R_{\mathrm{g}}=1.59 \mathrm{~nm}$ were calculated by fitting the SAXS pattern recorded for the PGMA $_{48}-$ PTFEMA $_{50}$ nanoparticles to a spherical micelle model; $^{36}$ this approach afforded a $\rho_{\text {particle }}$ of $1.15 \mathrm{~g} \mathrm{~cm}^{-3}$. The volume-average particle diameter, $D_{\mathrm{SAXS}}$, was calculated to be $20.0 \mathrm{~nm}$ using $D_{\text {SAXS }}=\left(2 R_{\mathrm{s}}+4 R_{\mathrm{g}}\right)$.

The effective density of the $n$-alkane droplets was estimated by first calculating the number of nanoparticles packed around a single droplet $(N)$.

$$
\begin{aligned}
& N=\frac{\text { total number of nanoparticles }}{\text { total number of droplets }} \\
& N=\frac{\left[m_{\text {particles }} N_{\mathrm{A}} / N_{\mathrm{s}} M_{\mathrm{n}}\right]}{\left[V_{\text {oil }} /\left(\frac{4}{3} \pi r_{\text {oil }}\right)^{3}\right]}
\end{aligned}
$$

Here, $m_{\text {particle }}$ is the mass of particles used to prepare the nanoemulsion, $N_{\mathrm{A}}$ is Avogadro's constant, and $N_{\mathrm{s}}$ is the number of $\mathrm{PGMA}_{48}-\mathrm{PTFEMA}_{50}$ chains per particle, determined by SAXS to be 139 using $N_{\mathrm{s}}=\frac{\frac{4}{3} \pi R_{\mathrm{s}}{ }^{3}}{V_{\text {PTFEMA }}}, M_{\mathrm{n}}$ is the number-average molecular mass of the PGMA $_{48}-$ PTFEMA $_{50}$ chains, $V_{\text {oil }}$ is the total volume of oil used to prepare each nanoemulsion, and $r_{\mathrm{oil}}$ is the radius of the minimum possible droplet diameter. We calculate $r_{\text {oil }}$ to be the mean DLS droplet radius $\left(r_{\mathrm{DLS}}\right)$ minus the adsorbed nanoparticle diameter to give the minimum bare oil droplet radius $\left(r_{\text {oil }}=r_{\mathrm{DLS}}-2 r_{\text {particle }}\right)$.

The PGMA $_{48}-$ PTFEMA $_{50}$ nanoparticle diameter $d$ was determined to be $20.0 \mathrm{~nm}$ by SAXS (where $d=2 R_{\mathrm{s}}+4 R_{\mathrm{g}}$ ). However, we consider the effective adsorbed diameter $\left(2 r_{\text {particle }}\right)$ at the oil/water interface to be given by $2 R_{\text {core }}+2 R_{\mathrm{g}}$ (or $16.8 \mathrm{~nm}$ ). This is because the $\mathrm{PGMA}_{48}$ stabilizer chains that are in direct contact with the oil phase are expected to be fully collapsed and hence should occupy negligible volume at the oil/water interface (Scheme 1). For example, a nanoemulsion droplet with an overall diameter of $220 \mathrm{~nm}$ should have a core droplet diameter of $220-(2 \times 16.8) \approx 186 \mathrm{~nm}$. Several assumptions are made in these calculations: (i) the $z$-average droplet diameter reported by DLS includes both the oil droplet and the nanoparticle shell and (ii) the nanoparticles adsorb at the $\mathrm{o} / \mathrm{w}$ interface with an effective contact angle of $0^{\circ}$ with respect to the nanoparticle core (see Scheme 1). Clearly, this is not the true nanoparticle contact angle; therefore, the droplet diameter will be slightly overestimated. However, this is likely to be a relatively small systematic error and therefore is not expected to affect the main findings of this study.

The effective density of each nanoemulsion droplet, $\rho_{\text {nanoemulsion }}$ was then calculated using a core-shell model where the core is composed of the $n$-alkane (whose density is denoted by $\rho_{\text {oil }}$ ) and the particulate shell comprises a monolayer of adsorbed $\mathrm{PGMA}_{48}$ PTFEMA $_{50}$ nanoparticles with an effective adsorbed particle radius $\left(r_{\text {particle }}\right)$ of $8.4 \mathrm{~nm}^{37}$ comprising the particulate shell. ${ }^{37}$
Scheme 1. Schematic Representation of the Adsorption of PGMA $_{48}-$ PTFEMA $_{50}$ Nanoparticles at the $n$-Alkane/Water Interface $^{a}$

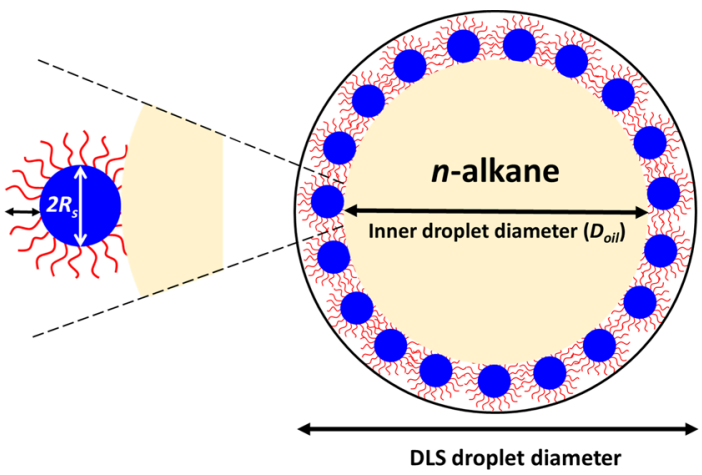

${ }^{a}$ It is assumed that (i) the nanoparticles are adsorbed with an effective contact angle of $0^{\circ}$ and (ii) $\mathrm{PGMA}_{48}$ stabilizer chains in direct contact with the $n$-alkane/water interface are fully collapsed and hence do not contribute to the adsorbed nanoparticle radius. Thus, given that the effective thickness of the adsorbed sterically stabilized nanoparticles is given by $2 R_{\mathrm{s}}+2 R_{\mathrm{g}}$ (rather than $2 R_{\mathrm{s}}+4 R_{\mathrm{g}}$ ), the approximate effective sphere radius, $r_{\text {particle }}$ of the adsorbed nanoparticles is given by $r_{\text {particle }}$ $=R_{\mathrm{s}}+R_{\mathrm{g}}$ or $8.4 \mathrm{~nm}$. Experimental values for $R_{\mathrm{s}}$ and $R_{\mathrm{g}}$ were obtained from SAXS analysis of the $\mathrm{PGMA}_{48}-\mathrm{PTFEMA}_{50}$ nanoparticles prior to emulsification, see the main text.

$$
\rho_{\text {nanoemulsion }}=\frac{N \rho_{\text {particle }} r_{\text {particle }}{ }^{3}+\rho_{\text {oil }} r_{\text {oil }}{ }^{3}}{N r_{\text {particle }}{ }^{3}+r_{\text {oil }}{ }^{3}}
$$

\section{RESULTS AND DISCUSSION}

The PGMA $_{48}-$ PTFEMA $_{50}$ nanoparticles used in this study were prepared as a $10 \%$ aqueous dispersion by RAFT aqueous emulsion polymerization using a technique known as polymerization-induced self-assembly (PISA), as reported previously (see Figure 1A). ${ }^{36}$ The $z$-average nanoparticle diameter was determined to be $24 \mathrm{~nm}$ by DLS, whereas fitting SAXS data to a spherical micelle model indicated a volume-average diameter of $20 \mathrm{~nm}$; TEM studies confirmed that the nanoparticles possessed a near-monodisperse spherical morphology (see Figure $1 \mathrm{~B}-\mathrm{D}$ ). Preliminary scoping experiments suggested that an excess of relatively small nanoparticles were required to maximize the total surface area available during the highpressure microfluidization processing step and hence ensure the formation of stable Pickering nanoemulsions. For example, utilizing somewhat larger PGMA $_{48}-$ PTFEMA $_{150}$ nanoparticles (51 nm diameter by DLS) merely produced highly flocculated nanoemulsions. ${ }^{34}$

Initially, a $7.0 \mathrm{w} / \mathrm{v} \%$ aqueous dispersion of $\mathrm{PGMA}_{48}$ PTFEMA $_{50}$ nanoparticles was used to prepare a relatively coarse Pickering macroemulsion with a mean droplet diameter of $40 \mu \mathrm{m}$ via conventional high-shear homogenization. Figure 2 illustrates how this precursor macroemulsion was then processed using the LV1 microfluidizer to produce a Pickering nanoemulsion. It is essential to have a substantial excess of PGMA $_{48}-$ PTFEMA $_{50}$ nanoparticles present after formation of the initial macroemulsion, as these non-adsorbed nanoparticles are required to stabilize the new oil/water interface generated during high-pressure microfluidization. In a recent related study, ${ }^{34}$ it was found empirically that an applied pressure of 20000 psi was optimal for the preparation of stable Pickering nanoemulsions. Lower pressures let to larger, more poly- 
<smiles>CC(C)(N)CC(C)(C)C(=O)OCC(O)CO</smiles>
$\mathrm{PGMA}_{48}$ macro CTA

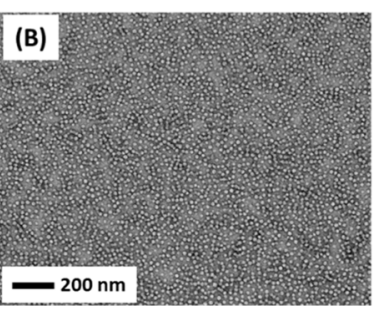

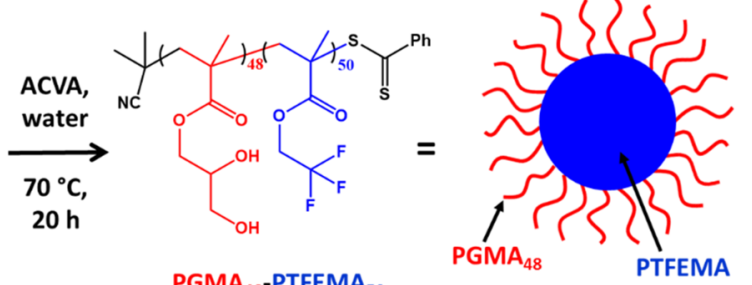

$\mathrm{PGMA}_{48}-\mathrm{PTFEMA}_{50}$
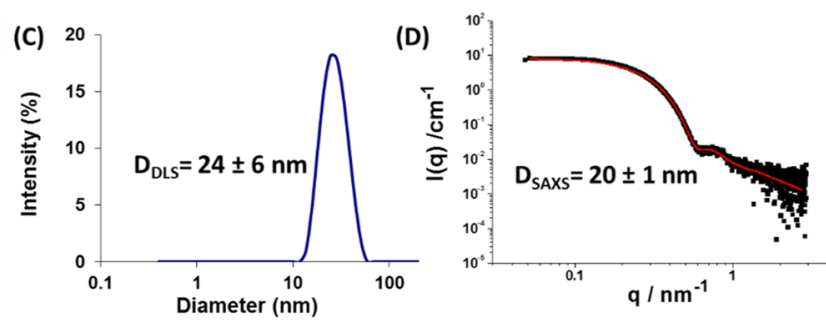

Figure 1. (A) Synthesis of $\mathrm{PGMA}_{48}-\mathrm{PTFEMA}_{50}$ nanoparticles via RAFT aqueous emulsion polymerization of TFEMA at $70{ }^{\circ} \mathrm{C}$ using a PGMA 48 macro-CTA; (B) TEM image of the resulting sterically stabilized PGMA $_{48}-$ PTFEMA $_{50}$ nanoparticles; (C) particle size distribution determined by DLS; (D) SAXS pattern recorded for a $1.0 \mathrm{w} / \mathrm{w} \%$ aqueous dispersion of the PGMA $_{48}-$ PTFEMA $_{50}$ nanoparticles (solid red line represents the data fit obtained using a spherical micelle model, which reports a volume-average diameter of $20 \pm 1 \mathrm{~nm}$ ).

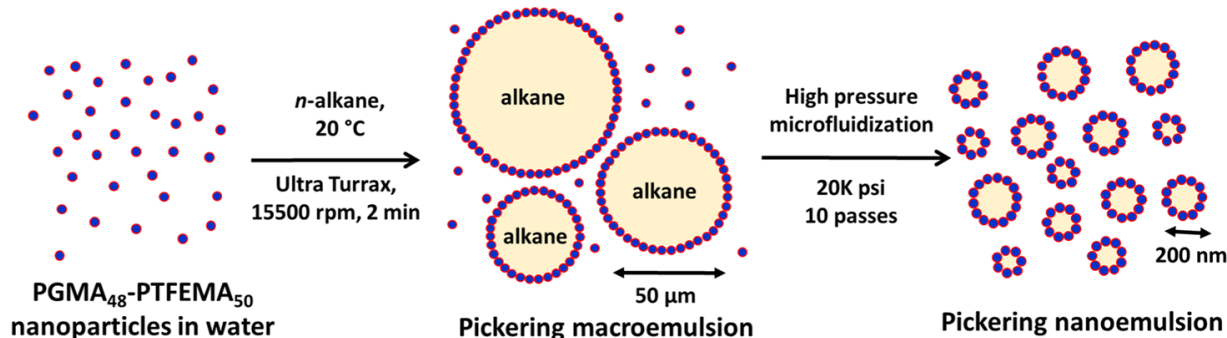

Figure 2. Schematic representation of the two-step preparation of Pickering nanoemulsions. First, a $7.0 \mathrm{w} / \mathrm{v} \%$ aqueous dispersion of PGMA 48 PTFEMA $_{50}$ nanoparticles were homogenized with one of four $n$-alkanes to form an oil-in-water Pickering macroemulsion of around $40-50 \mu \mathrm{m}$ diameter using conventional high-shear homogenization at $15500 \mathrm{rpm}$ for $2 \mathrm{~min}$ at $20^{\circ} \mathrm{C}$. This relatively coarse precursor emulsion was then refined via 10 passes through the LV1 microfluidizer at 20000 psi to obtain the final Pickering nanoemulsion.

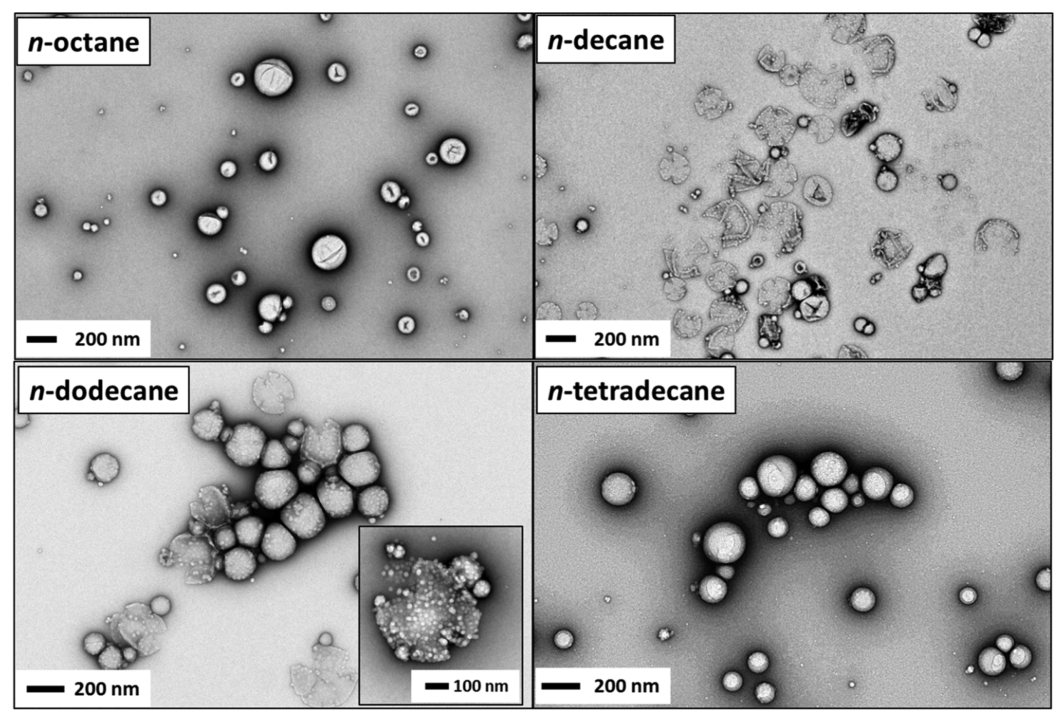

Figure 3. Representative TEM images obtained for freshly prepared Pickering nanoemulsions prepared via high-pressure microfluidization using $7.0 \mathrm{w} / \mathrm{w} \% \mathrm{PGMA}_{48}-\mathrm{PTFEMA}_{50}$ nanoparticles and $20 \mathrm{v} / \mathrm{v} \%$-alkane. Conditions: applied pressure $=20000$ psi for 10 passes using an LV1 microfluidizer.

disperse droplets, whereas higher pressures lead to the breakup of PGMA $_{48}-$ PTFEMA $_{50}$ nanoparticles into individual diblock copolymer chains, which can then act as an amphiphilic copolymer surfactant to form (non-Pickering) nanoemulsions.
Our previous studies focused on $n$-dodecane: for this oil, it was shown that at least eight passes through the microfluidizer were required to achieve a stable nanoemulsion with a unimodal droplet size distribution. ${ }^{34}$ Essentially the same 
Table 1. Summary of the Pickering Nanoemulsions Prepared Using Four $n$-Alkanes ( $n$-Octane, $n$-Decane, $n$-Dodecane, and $n$ Tetradecane $)^{a}$

\begin{tabular}{|c|c|c|c|c|c|c|c|}
\hline oil type & $\begin{array}{l}\text { initial DLS diameter, } \\
D_{\text {DLS }}(\mathrm{nm})^{b}\end{array}$ & $\begin{array}{l}\text { initial laser diffraction } \\
\quad \text { diameter, } D_{\mathrm{v}}(\mathrm{nm})\end{array}$ & $\begin{array}{c}\text { bare droplet } \\
\text { diameter, } D_{\text {oil }}(\mathrm{nm})^{c}\end{array}$ & $\begin{array}{l}\text { number of spheres } \\
\text { per droplet, } N\end{array}$ & $\begin{array}{c}\text { packing } \\
\text { efficiency (\%) }\end{array}$ & $\begin{array}{l}\rho_{n \text {-alkane }} \\
\left(\mathrm{g} \mathrm{cm}^{-3}\right)\end{array}$ & $\begin{array}{c}\rho_{\text {nanoemulsion }} \\
\left(\mathrm{g} \mathrm{cm}^{-3}\right)\end{array}$ \\
\hline n-octane & $179 \pm 59$ & $233 \pm 245$ & 145 & 120 & 44 & 0.70 & 0.77 \\
\hline$n$-decane & $182 \pm 56$ & $117 \pm 92$ & 148 & 128 & 45 & 0.73 & 0.80 \\
\hline n-dodecane & $257 \pm 93$ & $167 \pm 159$ & 223 & 438 & 74 & 0.75 & 0.81 \\
\hline$n$-tetradecane & $180 \pm 37$ & $120 \pm 95$ & 146 & 123 & 45 & 0.76 & 0.82 \\
\hline
\end{tabular}

${ }^{a}$ Droplet size distributions were recorded within $30 \mathrm{~min}$ of the preparation of each nanoemulsion. The SAXS-derived PGMA $48-\mathrm{PTFEMA}_{50}$ nanoparticle diameter, $D_{\mathrm{SAXS}}$, was determined to be $20.0 \mathrm{~nm}$ (where $D_{\mathrm{SAXS}}=2 R_{\text {core }}+4 R_{\mathrm{g}}$ ), whereas the effective adsorbed diameter at the oil/water interface was taken to be $2 R_{\text {core }}+2 R_{\mathrm{g}}(16.8 \mathrm{~nm})$ because the collapsed stabilizer chains that are in direct contact with the oil/water interface occupying a negligible volume. The effective nanoparticle density was estimated to be $1.15 \mathrm{~g} \mathrm{~cm}^{-3}$ using previously reported data. ${ }^{36} Z_{\text {-average }}$ diameter. ${ }^{c}$ Calculated using $D_{\text {oil }}=D_{\text {DLS }}-4 r_{\text {particle }}$.

observations were made for the other three $n$-alkanes selected for this study ( $n$-octane, $n$-decane, and $n$-tetradecane). These four oils were selected for their differing aqueous solubilities ${ }^{40,41}$ because this parameter is known to be important in determining the extent of Ostwald ripening for nanoemulsions. $^{42-44}$ After 10 passes through the LV1, a mean DLS droplet diameter of around $180 \mathrm{~nm}$ was obtained when using $n$-octane, $n$-decane, or $n$-tetradecane. In contrast, using $n$ dodecane produced a somewhat larger mean droplet diameter of $257 \mathrm{~nm}$ under the same conditions. The reason for this minor discrepancy is not known, but such experiments proved to be reproducible. It does not appear to be related to the mean diameter of the precursor Pickering macroemulsions, which was around $40 \mu \mathrm{m}$ for each of the four oils.

Transmission electron microscopy (TEM) images were obtained for dried fresh Pickering nanoemulsions, as shown in Figure 3. Although the volatile droplet phase is no longer present under the ultrahigh vacuum conditions required for TEM, some of the original superstructure of the adsorbed $\mathrm{PGMA}_{48}-\mathrm{PTFEMA}_{50}$ nanoparticles is preserved. Such postmortem studies provide convincing evidence that spherical droplets corresponding to nanoemulsion length scales were achieved when using each of the four oils, albeit with a significant degree of polydispersity. It is perhaps worth mentioning that the relatively high electron density of the PTFEMA cores provides relatively good contrast for such TEM studies, which is one advantage of using these particular amphiphilic PGMA $_{48}-$ PTFEMA $_{50}$ diblock copolymers.

Table 1 summarizes the initial droplet size distributions determined for Pickering nanoemulsions prepared using the four different $n$-alkanes. DLS and laser diffraction studies were conducted on fresh nanoemulsions within $30 \mathrm{~min}$ of their preparation. For three of the oils, the volume-average nanoemulsion diameters reported by laser diffraction are lower than the $z$-average diameters obtained from DLS, as expected. However, the DLS droplet diameter is actually lower than that reported by laser diffraction for the $n$-octane-based nanoemulsion. A possible explanation for this unexpected finding is that the latter nanoemulsion already exhibits signs of Ostwald ripening even at this rather early stage and that the DLS technique is surprisingly insensitive to the coarsening droplet size distribution (see the following discussion). The remarkably poor stability of this n-octane nanoemulsion is consistent with findings by Persson et al., ${ }^{21}$ who observed the appearance of significantly larger droplet diameters for the same oil after ageing a silica-stabilized nanoemulsion for just 30 $\min$.

Analytical centrifugation was used to further characterize both the initial and ageing nanoemulsions. This particular technique (LUMiSizer instrument) has a much higher resolution compared to DLS or laser diffraction because droplet fractionation occurs during the measurement (prior to detection). Thus, better insight into how the nanoemulsion size distributions change over time for each oil type was anticipated. One drawback of this technique is that the effective particle density is required to obtain an accurate particle size. ${ }^{36}$ This critical input parameter was estimated using eqs $1-3$ from the initial DLS droplet diameter using the additional data derived from the SAXS analysis of the $\mathrm{PGMA}_{48}-\mathrm{PTFEMA}_{50}$ nanoparticles (see the Experimental Section and the Supporting Information for details). The calculated nanoemulsion densities $\left(\rho_{\text {nanoemulsion }}\right)$ are shown in Table 1 for each $n$-alkane.

Another important consideration when using analytical centrifugation is the appropriate concentration at which the samples are analyzed. These Pickering nanoemulsions were prepared at a relatively high oil volume fraction $(20 \mathrm{v} / \mathrm{v} \% n$ alkane). However, analyzing droplets at such high concentrations is known to undersize substantially owing to the phenomenon of hindered sedimentation. ${ }^{45}$ In the present case, the rate of creaming of droplets within a relatively concentrated nanoemulsion when subjected to a centrifugal field is significantly retarded as a result of the close proximity of neighboring droplets; thus, the slower creaming rate leads to a smaller apparent droplet diameter. This artefact was indeed observed in the case of these Pickering nanoemulsions. Figure $4 \mathrm{~A}$ shows that, as the droplet concentration used for analytical centrifugation studies is systematically reduced, the apparent nanoemulsion droplet diameter increases and approaches a constant plateau value at approximately $1.0 \mathrm{v} / \mathrm{v} \%$. Thus, all further studies were conducted at this oil droplet concentration, with the $20 \mathrm{v} / \mathrm{v} \%$ stock nanoemulsion being diluted immediately prior to analysis. Using droplet concentrations lower than $1.0 \mathrm{v} / \mathrm{v} \%$ becomes problematic: such dilute emulsions scatter light only rather weakly and hence fall outside of the optimum transmission range required for the LUMiSizer instrument (i.e., below 30\% transmission). Figure $4 \mathrm{~B}$ illustrates the effect that varying the oil droplet concentration has on the apparent droplet size distributions reported by the instrument. Analyzing relatively concentrated nanoemulsions (diluted to only $10 \mathrm{v} / \mathrm{v} \%$ ) leads to a significantly smaller apparent droplet diameter (and an apparently narrower droplet size distribution).

Figure 5 summarizes the data obtained for various droplet characterization techniques for a freshly-made and 1-week-old $n$-octane-in-water Pickering nanoemulsion. A significant increase in the mean droplet diameter is discernible by laser diffraction (Figure 5A), analytical centrifugation (Figure 5B), 


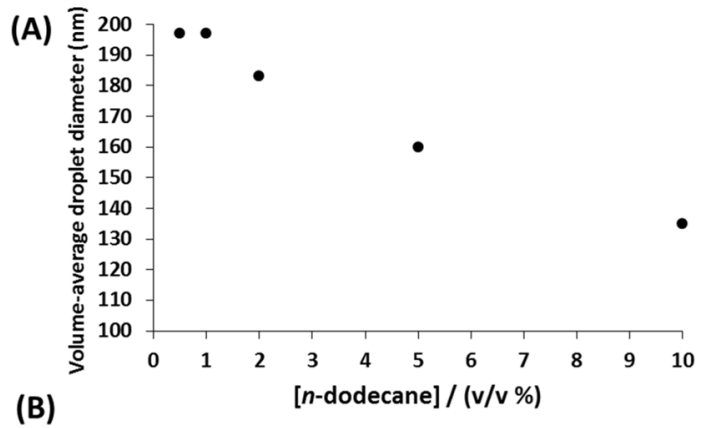

(B)

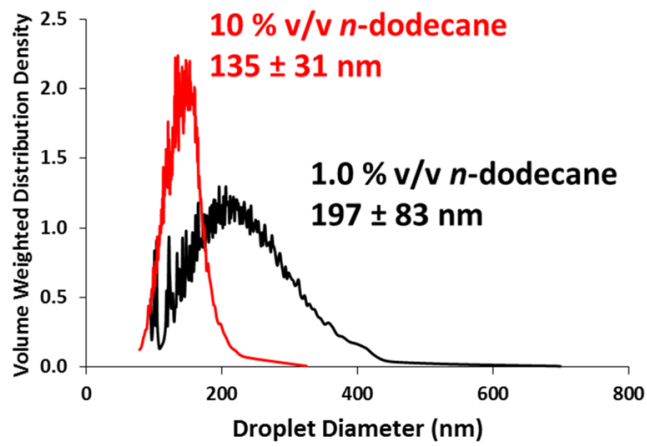

Figure 4. (A) Effect of droplet concentration on apparent droplet diameter for an $n$-dodecane-in-water Pickering nanoemulsion as determined by analytical centrifugation (LUMiSizer instrument). This hindrance function indicates that the optimum droplet concentration for such analyses is approximately $1.0 \mathrm{v} / \mathrm{v} \%$, with higher concentrations leading to hindered creaming and hence artefactual undersizing. (B) Droplet size distributions determined by analytical centrifugation using an effective nanoemulsion droplet density of 0.81 $\mathrm{g} \mathrm{cm}^{-3}$ at either 10 or $1.0 \mathrm{v} / \mathrm{v} \% n$-dodecane. As a result of these initial studies, all further LUMiSizer analyses were conducted at a droplet concentration of $1.0 \mathrm{v} / \mathrm{v} \% n$-alkane. and optical microscopy (Figure 5D) after ageing the $20 \mathrm{v} / \mathrm{v} \%$ nanoemulsion for 1 week at ambient temperature. Given the relatively high solubility of $n$-octane in water $\left(0.66 \mathrm{mg} \mathrm{dm}^{-3}\right.$ at $\left.20{ }^{\circ} \mathrm{C}\right),{ }^{40}$ such droplet growth is attributed to Ostwald ripening. ${ }^{42-44}$ Perhaps surprisingly, DLS analysis indicated only a minimal change in particle size: the $z$-average diameter actually decreased slightly from $179 \pm 59$ to $168 \pm 72 \mathrm{~nm}$, although a rather small population of micrometer-sized coarser droplets was also detected. However, the latter feature was only observed initially (see Figure 5C); it disappeared in subsequent runs (each measurement comprising an average of three runs). DLS is normally highly biased toward larger populations because the scattered light intensity scales according to the sixth power of the particle radius. This unexpected lack of sensitivity is puzzling, but one possible explanation could be the rapid creaming of the relatively large oil droplets (which were clearly detectable by laser diffraction and analytical centrifugation). This is consistent with the detection of large droplets during the first run but not during the two subsequent runs. Laser diffraction and analytical centrifugation studies indicated that a significant fraction of $n$ octane droplets ( $>60 \%$ according to analytical centrifugation) became micrometer-sized within 1 week of ageing at $20{ }^{\circ} \mathrm{C}$. This was confirmed by optical microscopy studies performed after 1 week (Figure 5D). According to the analytical centrifugation data, approximately $20 \%$ of the droplets had already grown to at least $1-2 \mu \mathrm{m}$ diameter by the time the fresh nanoemulsion was analyzed. This illustrates the high resolution of this technique: laser diffraction and DLS simply do not have the sensitivity to readily discriminate between a large population of submicrometer-sized droplets and a minor fraction of the micrometer-sized droplets. The former population cannot be observed by optical microscopy because

\section{A) Laser Diffraction}

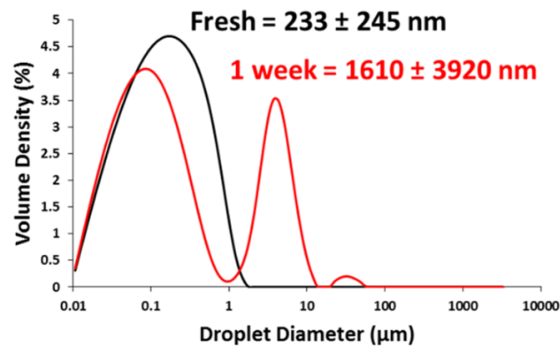

C) Analytical Centrifugation

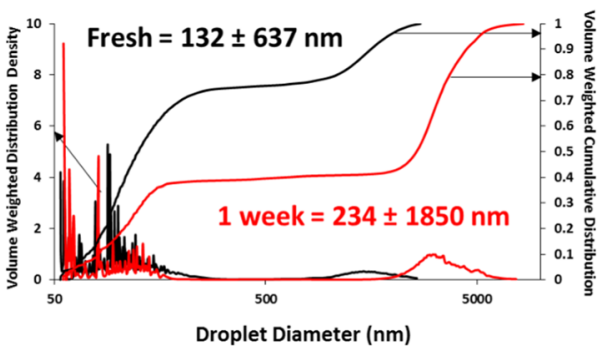

B) Dynamic Light Scattering

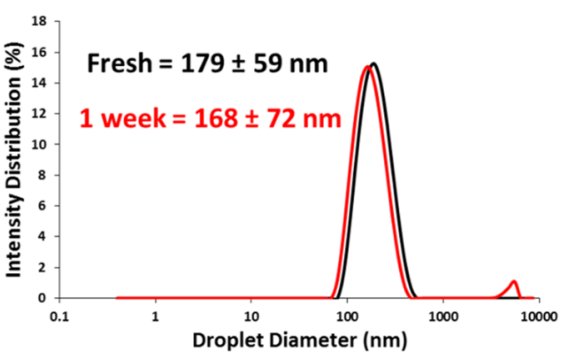

D) Optical Microscopy

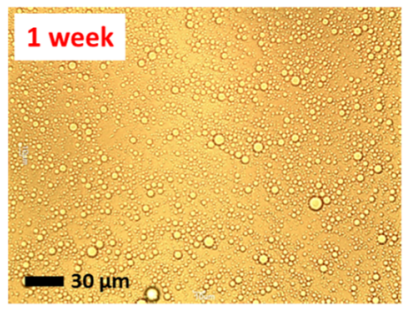

Figure 5. Various particle sizing techniques used to characterize a freshly-made and 1-week-old $20 \mathrm{v} / \mathrm{v} \% n$-octane-in-water Pickering nanoemulsion prepared using $7.0 \mathrm{w} / \mathrm{w} \% \mathrm{PGMA}_{48}-$ PTFEMA $_{50}$ nanoparticles at 20000 psi with 10 passes. (A) Laser diffraction, (B) DLS, (C) analytical centrifugation (LUMiSizer, $\rho_{\text {nanoemulsion }}=0.77 \mathrm{~g} \mathrm{~cm}^{-3}$ ), and (D) optical microscopy (no oil droplets were observed by optical microscopy for any of the freshly-made Pickering nanoemulsions reported in this study, but micrometer-sized oil droplets were clearly discernible within 1 week of ageing the $n$-octane-based Pickering nanoemulsion). Perhaps surprisingly, DLS seemed to be relatively insensitive to the larger oil droplets present within the 1-week-old Pickering nanoemulsion, whereas this coarse fraction was readily detected by laser diffraction and analytical centrifugation. 
Table 2. Change in Mean Droplet Diameter with Ageing Time as Determined by Analytical Centrifugation (LUMiSizer) for the Four $n$-Alkane Pickering Nanoemulsions Reported in This Study ${ }^{a}$

\begin{tabular}{|c|c|c|c|c|c|c|}
\hline \multirow[b]{2}{*}{ oil type } & \multirow[b]{2}{*}{ aqueous solubility of oil at $20^{\circ} \mathrm{C}\left(\mathrm{mg} \mathrm{dm}^{-3}\right)$} & \multirow[b]{2}{*}{$\rho_{\text {nanoemulsion }}\left(\mathrm{g} \mathrm{cm}^{-3}\right)$} & \multicolumn{4}{|c|}{ mean nanoemulsion diameter by analytical centrifugation $(\mathrm{nm})$} \\
\hline & & & fresh & 1 week & 4 weeks & 6 weeks \\
\hline n-octane & 0.66 & 0.77 & $132 \pm 637$ & $234 \pm 1850$ & & \\
\hline$n$-decane & 0.0396 & 0.80 & $115 \pm 219$ & $258 \pm 1108$ & & \\
\hline n-dodecane & 0.0034 & 0.81 & $160 \pm 92$ & $182 \pm 156$ & $186 \pm 227$ & $175 \pm 335$ \\
\hline$n$-tetradecane & 0.000386 & 0.82 & $145 \pm 67$ & $141 \pm 87$ & $146 \pm 146$ & $156 \pm 180$ \\
\hline
\end{tabular}

${ }^{a}$ All nanoemulsions were prepared with $20 \mathrm{v} / \mathrm{v} \% n$-alkane using $7.0 \mathrm{w} / \mathrm{w} \% \mathrm{PGMA}_{48}-\mathrm{PTFEMA}_{50}$ nanoparticles at 20000 psi after 10 passes through a LV1 high-pressure microfluidizer. Analytical centrifugation studies of the "fresh" nanoemulsions were conducted within $24 \mathrm{~h}$ of microfluidization in all cases. (N.B. significant evaporation of the $n$-octane- and $n$-decane-based nanoemulsions occurred within 1 week, which meant that no further analysis could be undertaken in these two cases).

such fine droplets are beyond the optical resolution limit. In the literature, nanoemulsions are typically sized using either DLS or laser diffraction, as such instruments are widely available in many research laboratories. In contrast, there are relatively few reports of sizing nanoemulsions via analytical centrifugation, yet this high-resolution technique appears to offer an important advantage when considering the effect of Ostwald ripening. For this reason, the rest of this article focuses on the characterization of Pickering nanoemulsions via analytical centrifugation. The caveat with this sizing technique is the uncertainty in calculating the effective droplet density for this particular nanoemulsion system and the technical problems associated with the superimposed density distribution on the droplet size distribution (see above).

Table 2 reports the mean volume-average diameter determined by analytical centrifugation for Pickering nanoemulsions prepared using each of the four $n$-alkanes on ageing at $20{ }^{\circ} \mathrm{C}$ for up to 6 weeks. Unfortunately, the relatively high volatilities of $n$-octane and $n$-decane meant that significant evaporation of the oil droplet phase occurred within 1 week, even if these nanoemulsions were well-sealed. Hence, it was not possible to accurately size these two nanoemulsions after ageing for 1 week. However, it is nevertheless evident that significant droplet coalescence occurred for these two nanoemulsions over this time frame compared to those prepared under the same conditions using $n$-dodecane or $n$ tetradecane. In particular, the width of the droplet size distributions observed for the fresh nanoemulsions became significantly broader from $n$-tetradecane to $n$-octane. For the two more volatile oils, these distributions become highly asymmetric, with pronounced tailing toward larger droplet diameters. This suggests that Ostwald ripening commences immediately after preparation of each nanoemulsion, with $n$ octane droplets coarsening significantly faster than $n$-decane droplets. After ageing for 1 week, only the $n$-tetradecane-based nanoemulsion retains its (approximate) original mean droplet diameter. The $n$-octane and $n$-decane nanoemulsions exhibited the largest increases in mean diameter, which correlate with the significantly higher aqueous solubilities of these two oils. All four droplet size distributions became broader, with the most pronounced change being observed for $n$-octane and only a minimal change being detected for $n$-tetradecane. Figure $6 \mathrm{~A}$ shows the volume-average cumulative distributions obtained for each of the four Pickering nanoemulsions recorded after ageing for 1 week. The extent of Ostwald ripening is clearly greatest for $n$-octane: around $60 \%$ of the droplets now exceed $500 \mathrm{~nm}$, with approximately $10 \%$ being larger than $4 \mu \mathrm{m}$. In comparison, the proportion of droplets exceeding $500 \mathrm{~nm}$ for the other three oils is $45 \%$ for $n$-decane, $9 \%$ for $n$-dodecane,
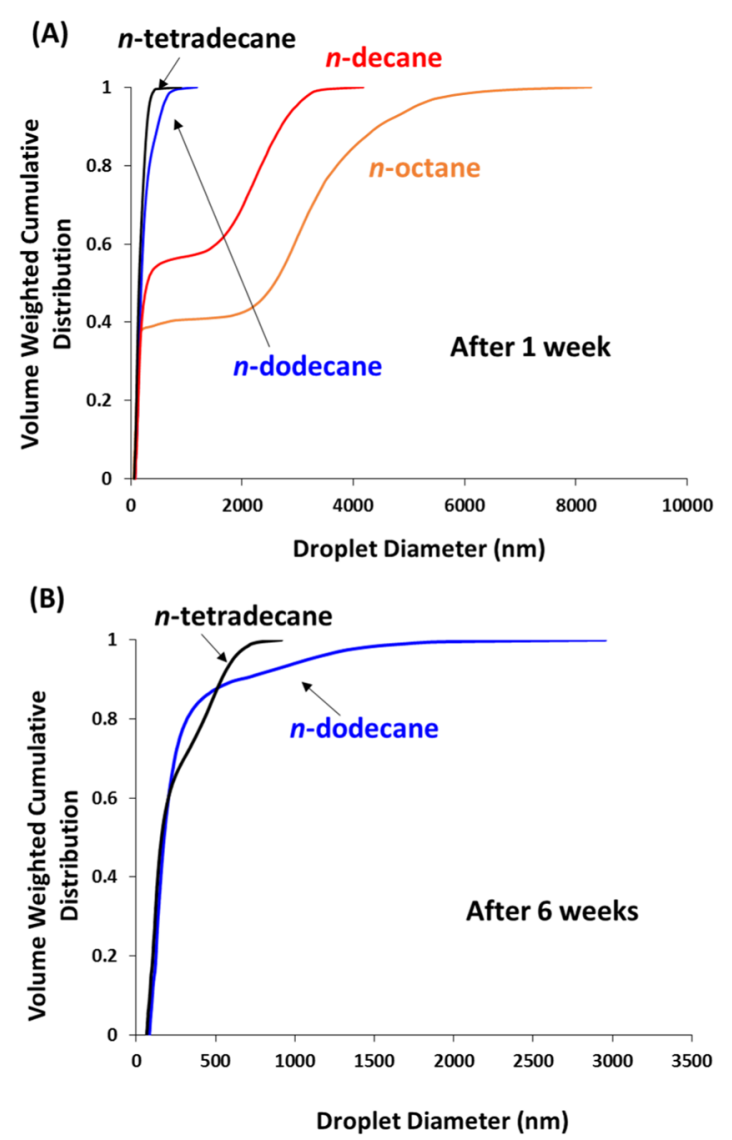

Figure 6. Volume-weighted cumulative distributions determined by analytical centrifugation (LUMiSizer instrument) for the $n$-alkane-inwater nanoemulsions: (A) after ageing for 1 week at $20^{\circ} \mathrm{C}$ and (B) after ageing for 6 weeks. Note that significant evaporation of the more volatile $n$-octane and $n$-decane oils occurred within 1 week; so, no further analysis was possible in these two cases.

but less than $0.1 \%$ for $n$-tetradecane. Although substantial Ostwald ripening had occurred within 1 week, significant proportions of the original $n$-octane $(>35 \%)$ and $n$-decane ( $>50 \%)$ formulations can still be classified as nanoemulsions. This represents much better stability compared to the silicastabilized nanoemulsions reported by Persson and co-workers. ${ }^{21}$ In principle, this difference may be simply related to the relatively large $20 \mathrm{~nm}$ PGMA $_{48}-\mathrm{PTFEMA}_{50}$ nanoparticles being more strongly adsorbed at the oil/water interface than the $7 \mathrm{~nm}$ charge-stabilized "hard sphere" silica nanoparticles utilized by the Swedish group. ${ }^{4}$ However, Vermant and coworkers have just reported that using sterically stabilized "soft 
spheres" leads to a much greater interfacial yield stress, which is believed to account for the enhanced long-term stability observed for many emulsions (and foams). ${ }^{46}$

After ageing for 4-6 weeks, the $n$-dodecane- and $n$ tetradecane-based nanoemulsions gradually undergo further Ostwald ripening, albeit to a much smaller extent than that observed for the $n$-octane and $n$-decane nanoemulsions. Analytical centrifugation studies indicate that the mean droplet diameter remains almost unchanged (Table 2), but the droplet distributions become significantly broader over time, with the $n$-dodecane-based nanoemulsion being prone to greater coarsening (with $6 \%$ of droplets exceeding $1 \mu \mathrm{m}$ diameter after 6 weeks) than that produced using the less water-soluble $n$-tetradecane. After 6 weeks ageing at $20^{\circ} \mathrm{C}$, all of the latter oil droplets remained below $1 \mu \mathrm{m}$ diameter, with around $65 \%$ still being classified as a nanoemulsion. In principle, at least some of this droplet coarsening could be attributed to a superimposed density distribution, as discussed earlier. ${ }^{36-38}$ However, in practice, this is likely to be a secondary effect. Overall, the Ostwald ripening observed for these Pickering nanoemulsions strongly correlate with the aqueous solubilities of the four $n$-alkanes used in this study. Given the bimodal size distributions observed for these Pickering nanoemulsions on ageing, it was not possible to examine whether the cube of the mean diameter for the ripening droplets depended linearly on the ageing time, as reported previously by Solans and coworkers. $^{47}$

\section{CONCLUSIONS}

In summary, a series of oil-in-water Pickering nanoemulsions have been prepared using four different $n$-alkanes stabilized by PGMA $_{48}-$ PTFEMA $_{50}$ nanoparticles. The Pickering nature of these nanoemulsions was confirmed by TEM studies because drying led to close-packed spherical superstructures comprising the original nanoparticles. Mean droplet diameters for the fresh nanoemulsions were determined by DLS, laser diffraction, and analytical centrifugation. Perhaps surprisingly, DLS proved to be inadequate for monitoring the Ostwald ripening of these Pickering nanoemulsions over time. This lack of sensitivity may be the result of rapid creaming of micrometer-sized droplets or it could simply reflect the relatively low upper size limit for this instrument. In contrast, laser diffraction was able to detect the growing population of larger oil droplets, but this technique suffered from relatively low resolution. Analytical centrifugation proved to be the most sensitive technique for studying droplet growth because the oil droplets are fractionated according to their size prior to detection. Timedependent studies using the latter technique indicated that the $n$-octane nanoemulsions were rather unstable as expected, given the relatively high aqueous solubility of this oil $(0.66 \mathrm{mg}$ $\left.\mathrm{dm}^{-3}\right)$. These droplets coarsened significantly on ageing for 1 week at $20^{\circ} \mathrm{C}$, with larger mean droplet diameters and a much broader droplet size distribution being obtained, as confirmed by optical microscopy studies. Nanoemulsions prepared using n-decane (aqueous solubility $=0.0396 \mathrm{mg} \mathrm{dm}^{-3}$ ) also proved to be relatively unstable on ageing. In contrast, nanoemulsions prepared using either $n$-dodecane or $n$-tetradecane were significantly more stable, as expected given their much lower aqueous solubilities $\left(0.0034\right.$ and $0.000386 \mathrm{mg} \mathrm{dm}^{-3}$, respectively). After ageing for 6 weeks at $20^{\circ} \mathrm{C}$, only minimal change in the mean droplet diameter was observed for these latter two oils, but significant broadening of their respective droplet size distributions was discernible. Nevertheless, $94 \%$ of $n$-dodecane droplets and $100 \%$ of $n$-tetradecane droplets remained below $1 \mu \mathrm{m}$ diameter after this ageing period.

\section{ASSOCIATED CONTENT}

\section{S Supporting Information}

The Supporting Information is available free of charge on the ACS Publications website at DOI: 10.1021/acs.langmuir.8b01835.

Details of the spherical micelle models used for the SAXS analysis (PDF)

\section{AUTHOR INFORMATION}

\section{Corresponding Authors}

*E-mail: kate.thompson@manchester.ac.uk (K.L.T.).

*E-mai: s.p.armes@shef.ac.uk (S.P.A.).

ORCID

Matthew J. Derry: 0000-0001-5010-6725

Fiona L. Hatton: 0000-0002-0105-7530

Steven P. Armes: 0000-0002-8289-6351

Notes

The authors declare no competing financial interest.

\section{ACKNOWLEDGMENTS}

S.P.A. thanks the financial support from EPSRC (Fellowship grant EP/R003009/1) and also acknowledges a five-year Advanced Investigator ERC grant (PISA 320372).

\section{REFERENCES}

(1) Pickering, S. U. CXCVI.-Emulsions. J. Chem. Soc. 1907, 91, 2001-2021.

(2) Ramsden, W. Separation of solids in the surface-layers of solutions and "suspensions" (observations on surface-membranes, bubbles, emulsions, and mechanical coagulation)-Preliminary account. Proc. R. Soc. London 1903, 72, 156-164.

(3) Thompson, K. L.; Fielding, L. A.; Mykhaylyk, O. O.; Lane, J. A.; Derry, M. J.; Armes, S. P. Vermicious thermo-responsive Pickering emulsifiers. Chem. Sci. 2015, 6, 4207-4214.

(4) Binks, B. P. Particles as surfactants - similarities and differences. Curr. Opin. Colloid Interface Sci. 2002, 7, 21-41.

(5) Binks, B. P.; Lumsdon, S. O. Pickering emulsions stabilized by monodisperse latex particles: Effects of particle size. Langmuir 2001, $17,4540-4547$.

(6) Binks, B. P.; Lumsdon, S. O. Stability of oil-in-water emulsions stabilised by silica particles. Phys. Chem. Chem. Phys. 1999, 1, 30073016.

(7) Fujii, S.; Randall, D. P.; Armes, S. P. Synthesis of polystyrene/ poly2-(dimethylamino) ethyl methacrylate-stat-ethylene glycol dimethacrylatel core-shell latex particles by seeded emulsion polymerization and their application as stimulus-responsive particulate emulsifiers for oil-in-water emulsions. Langmuir 2004, 20, 1132911335 .

(8) Kalashnikova, I.; Bizot, H.; Cathala, B.; Capron, I. New Pickering emulsions stabilized by bacterial cellulose nanocrystals. Langmuir 2011, 27, 7471-7479.

(9) Nguyen, B. T.; Wang, W.; Saunders, B. R.; Benyahia, L.; Nicolai, T. pH-Responsive water-in-water Pickering emulsions. Langmuir 2015, 31, 3605-3611.

(10) Nicolai, T.; Murray, B. Particle stabilized water in water emulsions. Food Hydrocolloids 2017, 68, 157-163.

(11) Rizzelli, S. L.; Jones, E. R.; Thompson, K. L.; Armes, S. P. Preparation of non-aqueous Pickering emulsions using anisotropic block copolymer nanoparticles. Colloid Polym. Sci. 2016, 294, 1-12.

(12) Thompson, K. L.; Lane, J. A.; Derry, M. J.; Armes, S. P. Nonaqueous Isorefractive Pickering Emulsions. Langmuir 2015, 31, 43734376. 
(13) Fielding, L. A.; Armes, S. P. Preparation of Pickering emulsions and colloidosomes using either a glycerol-functionalised silica sol or core-shell polymer/silica nanocomposite particles. J. Mater. Chem. 2012, 22, 11235-11244.

(14) Ikem, V. O.; Menner, A.; Bismarck, A. High-porosity macroporous polymers sythesized from titania-particle-stabilized medium and high internal phase emulsions. Langmuir 2010, 26, $8836-8841$.

(15) Cui, Y.; Threlfall, M.; van Duijneveldt, J. S. Optimizing organoclay stabilized Pickering emulsions. J. Colloid Interface Sci. 2011, 356, 665-671.

(16) Cui, Y.; van Duijneveldt, J. S. Adsorption of polyetheramines on montmorillonite at high $\mathrm{pH}$. Langmuir 2010, 26, 17210-17217.

(17) Cui, Y.; van Duijneveldt, J. S. Microcapsules composed of crosslinked organoclay. Langmuir 2012, 28, 1753-1757.

(18) Williams, M.; Armes, S. P.; York, D. W. Clay-based colloidosomes. Langmuir 2012, 28, 1142-1148.

(19) Katepalli, H.; John, V. T.; Bose, A. The response of carbon black stabilized oil-in-water emulsions to the addition of surfactant solutions. Langmuir 2013, 29, 6790-6797.

(20) Gautier, F.; Destribats, M.; Perrier-Cornet, R.; Dechézelles, J.F.; Giermanska, J.; Héroguez, V.; Ravaine, S.; Leal-Calderon, F.; Schmitt, V. Pickering emulsions with stimulable particles: from highlyto weakly-covered interfaces. Phys. Chem. Chem. Phys. 2007, 9, 64556462.

(21) Persson, K. H.; Blute, I. A.; Mira, I. C.; Gustafsson, J. Creation of well-defined particle stabilized oil-in-water nanoemulsions. Colloids Surf., A 2014, 459, 48-57.

(22) Sihler, S.; Schrade, A.; Cao, Z.; Ziener, U. Inverse Pickering emulsions with droplet sizes below $500 \mathrm{~nm}$. Langmuir 2015, 31, 10392-10401.

(23) Sacanna, S.; Kegel, W. K.; Philipse, A. P. Thermodynamically stable Pickering emulsions. Phys. Rev. Lett. 2007, 98, 158301.

(24) Solans, C.; Izquierdo, P.; Nolla, J.; Azemar, N.; Garcia-Celma, M. J. Nano-emulsions. Curr. Opin. Colloid Interface Sci. 2005, 10, $102-110$.

(25) Sonneville-Aubrun, O.; Simonnet, J.-T.; L'Alloret, F. Nanoemulsions: a new vehicle for skincare products. Adv. Colloid Interface Sci. 2004, 108, 145-149.

(26) Rapoport, N. Y.; Kennedy, A. M.; Shea, J. E.; Scaife, C. L.; Nam, K.-H. Controlled and targeted tumor chemotherapy by ultrasound-activated nanoemulsions/microbubbles. J. Controlled Release 2009, 138, 268-276.

(27) McClements, D. J.; Rao, J. Food-grade nanoemulsions: Formulation, fabrication, properties, performance, biological fate, and potential toxicity. Crit. Rev. Food Sci. Nutr. 2011, 51, 285-330.

(28) Du, Z.; Wang, C.; Tai, X.; Wang, G.; Liu, X. Optimization and characterization of biocompatible oil-in-water nanoemulsion for pesticide delivery. ACS Sustain. Chem. Eng. 2016, 4, 983-991.

(29) Teo, A.; Goh, K. K. T.; Wen, J.; Oey, I.; Ko, S.; Kwak, H.-S.; Lee, S. J. Physicochemical properties of whey protein, lactoferrin and Tween 20 stabilised nanoemulsions: Effect of temperature, $\mathrm{pH}$ and salt. Food Chem. 2016, 197, 297-306.

(30) Wooster, T. J.; Golding, M.; Sanguansri, P. Impact of oil type on nanoemulsion formation and ostwald ripening stability. Langmuir 2008, 24, 12758-12765.

(31) Gutiérrez, J. M.; González, C.; Maestro, A.; Solé, I.; Pey, C. M.; Nolla, J. Nano-emulsions: New applications and optimization of their preparation. Curr. Opin. Colloid Interface Sci. 2008, 13, 245-251.

(32) Kentish, S.; Wooster, T. J.; Ashokkumar, A.; Balachandran, S.; Mawson, R.; Simons, L. The use of ultrasonics for nanoemulsion preparation. Innovat. Food Sci. Emerg. Technol. 2008, 9, 170-175.

(33) Jiménez Saelices, C.; Capron, I. Design of Pickering micro and nanoemulsions based on the structural characteristics of nanocelluloses. Biomacromolecules 2018, 19, 460.

(34) Thompson, K. L.; Cinotti, N.; Jones, E. R.; Mable, C. J.; Fowler, P. W.; Armes, S. P. Bespoke diblock copolymer nanoparticles enable the production of relatively stable oil-in-water Pickering nanoemulsions. Langmuir 2017, 33, 12616-12623.
(35) This overall diameter of $220 \mathrm{~nm}$ necessarily includes the thickness of the adsorbed PGMA48-PTFEMA50 nanoparticle layer. If this contribution is subtracted from the overall droplet diameter, the mean diameter for the underlying "naked" oil droplet is less than 200 $\mathrm{nm}$, which meets the criterion for a nanoemulsion according to the literature (see ref 24).

(36) Akpinar, B.; Fielding, L. A.; Cunningham, V. J.; Ning, Y.; Mykhaylyk, O. O.; Fowler, P. W.; Armes, S. P. Determining the effective density and stabilizer layer thickness of sterically stabilized nanoparticles. Macromolecules 2016, 49, 5160-5171.

(37) Fielding, L. A.; Mykhaylyk, O. O.; Armes, S. P.; Fowler, P. W.; Mittal, V.; Fitzpatrick, S. Correcting for a density distribution: Particle size analysis of core-shell nanocomposite particles using disk centrifuge photosedimentometry. Langmuir 2012, 28, 2536-2544.

(38) For the present nanoemulsion system, the core comprises a low-density oil and the shell is composed of high-density nanoparticles. However, in this particular case the overall droplet density is less than that of the continuous phase, so centrifugation leads to creaming rather than sedimentation. Thus artefactual broadening of the droplet size distribution is anticipated, rather than artefactual narrowing.

(39) Ilavsky, J.; Jemian, P. R. Irena: tool suite for modeling and analysis of small-angle scattering. J. Appl. Crystallogr. 2009, 42, 347353.

(40) McAuliffe, C. Solubility in water of C1-C9 hydrocarbons. Nature 1963, 200, 1092.

(41) Taylor, P. Ostwald ripening in emulsions: estimation of solution thermodynamics of the disperse phase. Adv. Colloid Interface Sci. 2003, 106, 261-285.

(42) Binks, B. P.; Cho, W.-G.; Fletcher, P. D. I.; Petsev, D. N. Stability of oil-in-water emulsions in a low interfacial tension system. Langmuir 2000, 16, 1025-1034.

(43) Binks, B. P.; Clint, J. H.; Fletcher, P. D. I.; Rippon, S.; Lubetkin, S. D.; Mulqueen, P. J. Kinetics of swelling of oil-in-water emulsions. Langmuir 1998, 14, 5402-5411.

(44) Binks, B. P.; Clint, J. H.; Fletcher, P. D. I.; Rippon, S.; Lubetkin, S. D.; Mulqueen, P. J. Kinetics of swelling of oil-in-water emulsions stabilized by different surfactants. Langmuir 1999, 15, 4495-4501.

(45) Walter, J.; Thajudeen, T.; Sü $\beta$, S.; Segets, D.; Peukert, W. New possibilities of accurate particle characterisation by applying direct boundary models to analytical centrifugation. Nanoscale 2015, 7, $6574-6587$.

(46) Beltramo, P. J.; Gupta, M.; Alicke, A.; Liascukiene, I.; Gunes, D. Z.; Baroud, C. N.; Vermant, J. Arresting dissolution by interfacial rheology design. Proc. Natl. Acad. Sci. U.S.A. 2017, 114, 10373.

(47) Izquierdo, P.; Esquena, J.; Tadros, T. F.; Dederen, C.; Garcia, M. J.; Azemar, N.; Solans, C. Formation and stability of nanoemulsions prepared using the phase inversion temperature method. Langmuir 2002, 18, 26-30. 